\title{
Chronisch entzündliche Darmerkrankungen - Standards und Ausblick
}

Die letzten Jahre haben erstaunliche Fortschritte in der Aufklärung von Ätiologie und Pathogenese chronisch entzündlicher Darmerkrankungen gebracht. Insbesondere die Entdeckung des ersten Gens, dessen Mutation bei 15-20\% der Patienten einen Morbus Crohn (abhängig von der Lebensregion) hervorruft, hat jahrelange Bemühungen Früchte tragen lassen. Auch die gestörten Interaktionen von Bakterien und dem intestinalen Epithel sind in ihrer Bedeutung besser verstanden worden und haben zu ersten therapeutischen Ansätzen geführt. Die Tatsache, dass das Auftreten des Morbus Crohn nur bei einem Teil der Patienten durch die erwähnte Mutation erklärbar ist, hat ebenso wie die klinische Beobachtung unterschiedlicher Phänotypen zur Folge, dass die Existenz zweier umschriebener Krankheiten im Rahmen der entzündlichen Darmerkrankungen inzwischen bezweifelt wird und Experten davon ausgehen, dass es sich um zwei Syndrome handelt, die aus zahlreichen Patientengruppen unterschiedlichen Phänotyps und vermutlich auch unterschiedlichen Genotyps zusammengesetzt sind. Daraus haben sich erste Hinweise für differenzierte Therapienotwendigkeiten und Prognose ergeben.

Die Entwicklung zahlreicher neuer bildgebender Verfahren wie beispielsweise die Dünndarmdarstellung mit Hilfe der Kernspinresonanztomographie, die dreidimensionale Rekonstruktion des abdominalen Inhalts und eventuell auch die Kapselendoskopie machen es erforderlich, die verfügbaren diagnostischen Methoden gezielter und den jeweiligen Fragestellungen angepasst einzusetzen. Neue Laborverfahren, insbesondere unterschiedliche Antikörper, haben ebenfalls einen Platz in der Diagnostik gefunden.

Das Karzinomrisiko bei Colitis ulcerosa ist abhängig von der Laufzeit und von der Ausdehnung der Erkrankung. Neuere Daten weisen darauf hin, dass offenbar auch die Konsequenz und die Art der langfristigen Therapie hier eine wesentliche Rolle spielen. Bei konsequenter remissionserhaltender Behandlung mit 5-ASA ist ebenso wie bei engagierter chirurgischer Behandlung nicht mit einem erhöhten Karzinomrisiko zu rechnen, wie Daten aus Dänemark und Großbritannien zeigen.

In der konservativen Standardtherapie des Morbus Crohn stehen nach wie vor die Glukokortikosteroide im Vordergrund. Die über lange Jahre zu zurückhaltend eingesetzte Immunsuppression, beispielsweise mit Azathioprin, wird vermehrt angewandt. Auch die 5-ASA-Präparate und nichtsystemische Steroide wie Budesonid haben ihren Platz, insbesondere in der breiten Anwendung, da neuere Daten zeigen, dass in einer populationsbezogenen Kohorte nicht einmal die Hälfte der Patienten jemals Steroide benötigte.

Bei Colitis ulcerosa dominieren die 5-ASA-Präparate in der Standardtherapie, bei einer kleineren Gruppe von Patienten werden Steroide und bei einigen auch Immunsuppressiva wie Ciclosporin oder Azathioprin als Langzeittherapie eingesetzt. Auch die Chirurgie hat ihren Stellenwert. Neue Therapieansätze beinhalten Probiotika, Antibiotika und die so genannten biologischen Therapien, von denen bislang nur Infliximab, ein Tumornekrosefaktor-Antikörper, zugelassen ist. Dessen Indikation beschränkt sich auf Patienten, die mit den genannten Therapiemaßnahmen nicht erfolgreich behandelt werden können. Bezüglich der Fisteln wird auch ein chirurgischer Versuch gefordert.

Die Operationstechniken haben sich in den letzten Jahren nicht wesentlich gewandelt. Bei der Colitis ulcerosa dominieren die Pouch-Verfahren, obwohl Patientenbefragungen auch ein hohes $\mathrm{Maß}$ an Zufriedenheit nach terminalem Stoma ergeben haben. Bei Morbus Crohn besteht für die darmsparende Operationstaktik einschließlich der Strikturoplastiken nach wie vor Konsens. Von Interesse ist die Neuentwicklung laparoskopischer Operationsformen, die zunehmend an Bedeutung gewinnen.

Ein wesentliches Problem nach Kolektomie und Pouch-Anlage stellt die Pouchitis dar. Hier handelt es sich offensichtlich um ein spezifisches Problem bei Patienten mit chronisch entzündlichen Darmerkrankungen, da die Pouchitis bei Patienten nach Kolektomie wegen einer familiären adenomatösen

\section{KARGER}

Fax +49761 4520714

E-mail Information@Karger.de

www.karger.com

(C) 2002 S. Karger GmbH, Freiburg 
Polyposis praktisch nicht beobachtet wird. Man unterscheidet die akute und die chronische Pouchitis, wobei die Behandlung der akuten Pouchitis einfacher ist und durch Antibiotika sowie gelegentlich eine lokale Therapie mit 5-ASA und Steroiden erfolgen kann, während die chronische Pouchitis deutlich schwieriger zu therapieren ist und gelegentlich auch eine Pouch-Entfernung erfordert.

Nach wie vor wird die Bedeutung von Mangelzuständen und insbesondere der Knochenstoffwechselstörungen, die erkannt und auch prophylaktisch behandelt werden müssen, unterschätzt.
Insgesamt haben die Entwicklungen der letzten Jahrzehnte die Überlebenswahrscheinlichkeit der Patienten praktisch normalisiert. Die Lebensqualität ist durch Optimierung der konservativen und der chirurgischen Therapie zwar besser, aber noch nicht optimal geworden. Hier liegt der Ansatzpunkt für die neuen Therapieverfahren, die bei zunehmender Kenntnis der Ätiopathogenese auch eine Heilung der mukosalen Veränderungen zum Ziel haben. Es bleibt abzuwarten, wie rasch die weitere Aufklärung der genetischen Grundlagen und damit verbunden die möglichen therapeutischen Neuentwicklungen vonstatten gehen werden.

J. Schölmerich, Regensburg 